\title{
consolidation dynamique des fondations du barrage de Pénitas, au Mexique
}

\section{dynamic consolidation of the foundation bed for the proposed Penitas Dam in Mexico}

\author{
M. GAMBIN \\ Directeur du département consolidations des sols, Soletanche Entreprise*
}

\begin{abstract}
Résumé
La consolidation dynamique, en accroîssant la densité relative du sol et le coefficient de pression horizontale des terres au repos $\mathrm{K}_{\mathrm{o}}$ d'une part, et en provoquant un vieillissement sismique du terrain grâce aux cycles répétés de liquéfaction drainage qui sont le principe même de la méthode, d'autre part, permet de diminuer le potentiel de liquéfication des formations naturelles ou des rembiais saturés en cas de tremblement de terre.
\end{abstract}

A l'occasion de la construction du barrage hydro-électrique de Pénitas sur le fleuve Grijalva, dans l'Eat de Chiapas au Mexique (zone de haute sismicité), la reconnaissance des sols ayant mis en évidence des densités relatives très variables, le Maître d'Ouvrage (la Commission Fédérale de l'Electricité) a décidé de procéder à l'amélioration et à l'homogénéisation des caractéristiques des alluvions ainsi que celles du remblai qui permettait de mettre le chantier au-dessus des plus hautes eaux.

L'objet de la communication est de présenter les résultats de la consolidation dynamique Ménard, appliquée à une couche de sol de $15 \mathrm{~m}$ d'épaisseur.

\section{Abstract}

Dynamic consolidation increases both the relative density of the soil and the horizontal pressure at rest factor $K_{0}$. Also this technique induces a seismic aging of the soil by the repeated cycles of liquefaction - drainage which makes the core of the treatment process. Consequently there is no wonder why this technique can be used to decrease the liquefaction potential of natural formations in earthquake prone areas.

In the Chiapas State, where seismicity level is rather high, the proposed Penitas Dam brings to completion the Grijalva river electric scheme.

Prior to its construction, the routine soil investigation showed large variations in the relative density of the alluvium. The Owner (the Federal Commission for Electricity) concluded that it was necessary to improve the alluvium parameters and decrease their variability. The treatment had also to be applied to the upper landfill which was designed to put the working platform above highest water level.

The aim of this paper is to submit the results of the Menard Dynamic Consolidation successfully applied to a soil layer $15 \mathrm{~m}$ thick.

* 6, rue de Watford, 92000 Nanterre. 


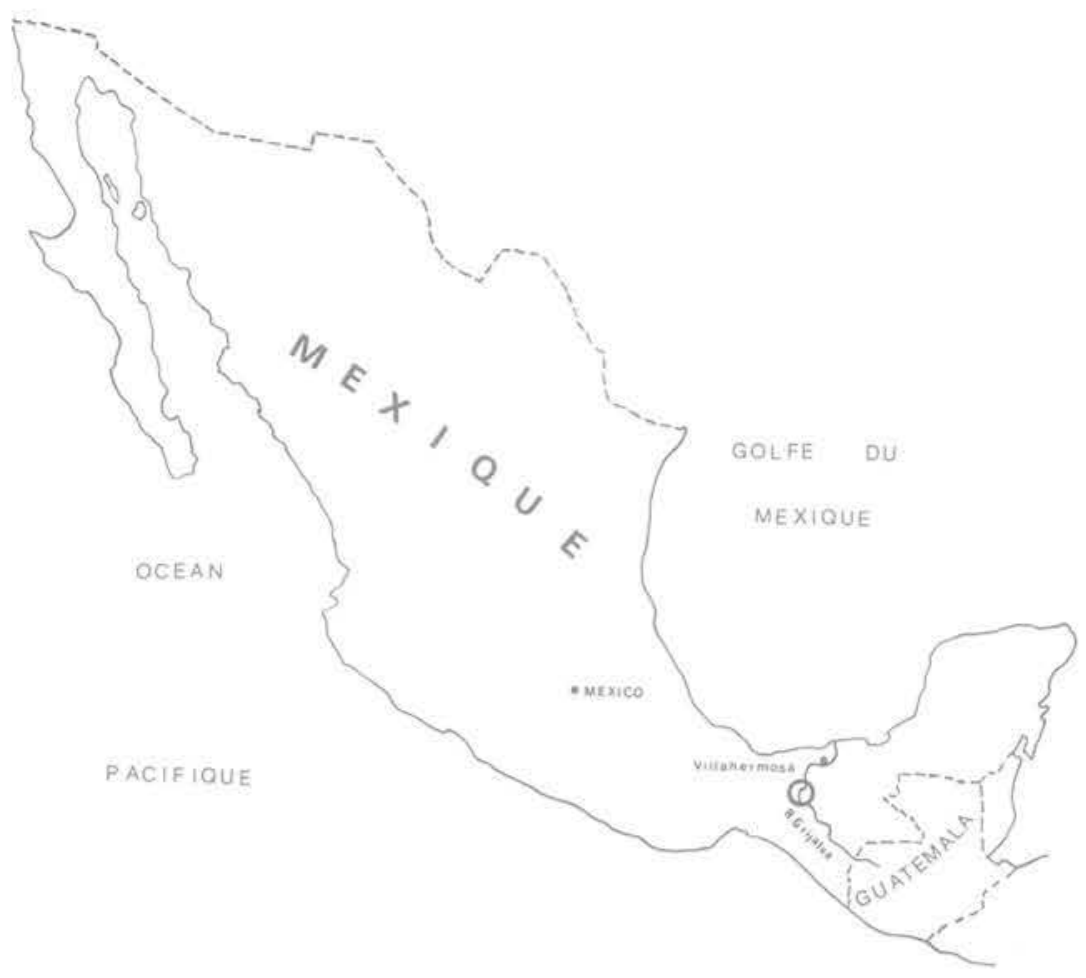

Fig. 1. - Localisation du projet.

La phase finale de l'équipement hydro-électrique du fleuve Grijalva, dans le sud du Mexique, à l'amont de Villahermosa (fig. 1) consiste en l'aménagement du site de Pénitas, dans l'état de Chiapas, $50 \mathrm{~km}$ à l'aval du barrage de Netzahuacoyotl déjà en service.

La figure 2 donne la coupe géologique en travers du fleuve qui comprend deux bras dans cette zone.

La figure 3 donne une vue en plan du projet dont la coupe AA est reportée sur la figure 4. La Commission Fédérale de l'Electricité (C.F.E.) avait finalement retenu la solution d'un barrage en terre de $44 \mathrm{~m}$ de hauteur, de $580 \mathrm{~m}$ en crête et de $200 \mathrm{~m}$ à $300 \mathrm{~m}$ de largeur à la base. Il s'agit d'un barrage composite dont le noyau central est prolongé dans les alluvions par une paroi plastique. On notera que les batardeaux amont et aval nécessaires pour assurer le détournement des eaux du fleuve pendant les travaux sont incorporés à l'ouvrage final.

\section{RÉSULTATS DE L'ÉTUDE DES SOLS PRÉLIMINAIRES}

L'étude des sols formant le remplissage de la vallée fut exécutée à l'aide d'essais de pénétration standard (S.P.T.) et d'essais de perméabilité Lefranc. Une coupe typique de sondage est représentée sur la figure 5 . On constate que les alluvions constituent une couche d'une cinquantaine de mètres d'épaisseur au maximum, dont la résistance à la pénétration dynamique est très variable, avec des faiblesses $(N<15)$ jusqu'à $15 \mathrm{~m}$ de profondeur, et que la perméabilité est très variable. Ces alluvions sont essentiellement composées de sables fins à moyens, avec un faible pourcentage de silt et des lentilles de graviers pouvant contenir des cailloux de $30 \mathrm{~cm}$ de grosseur. La figure 6 présente quelques courbes granulométriques types et leur fuseau enveloppe.

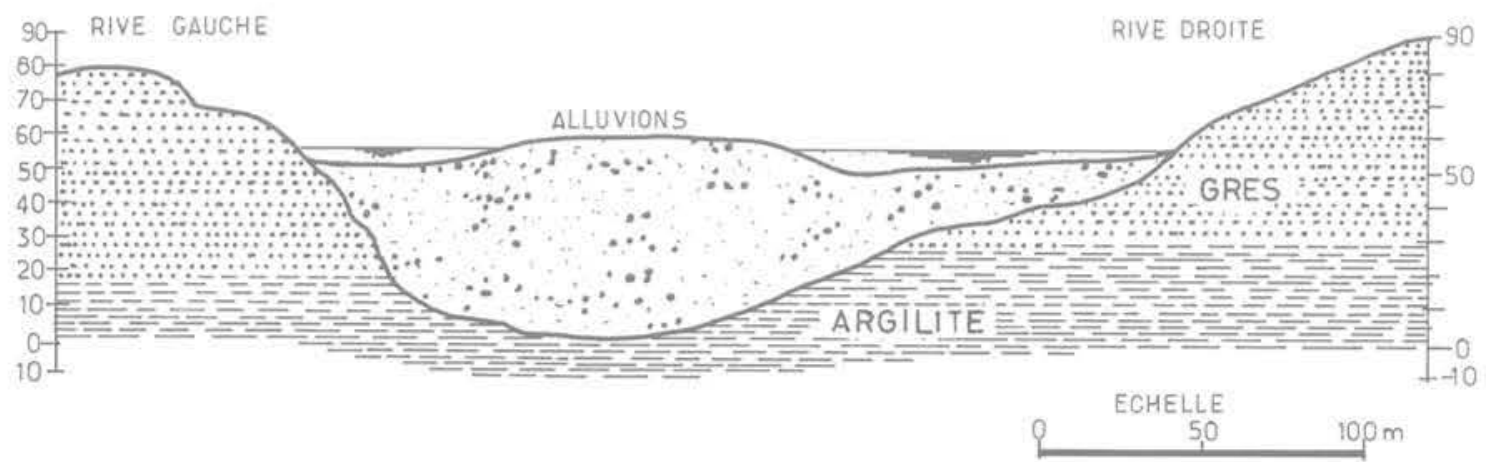

Fig. 2. - Coupe en travers du lit du fleuve. 


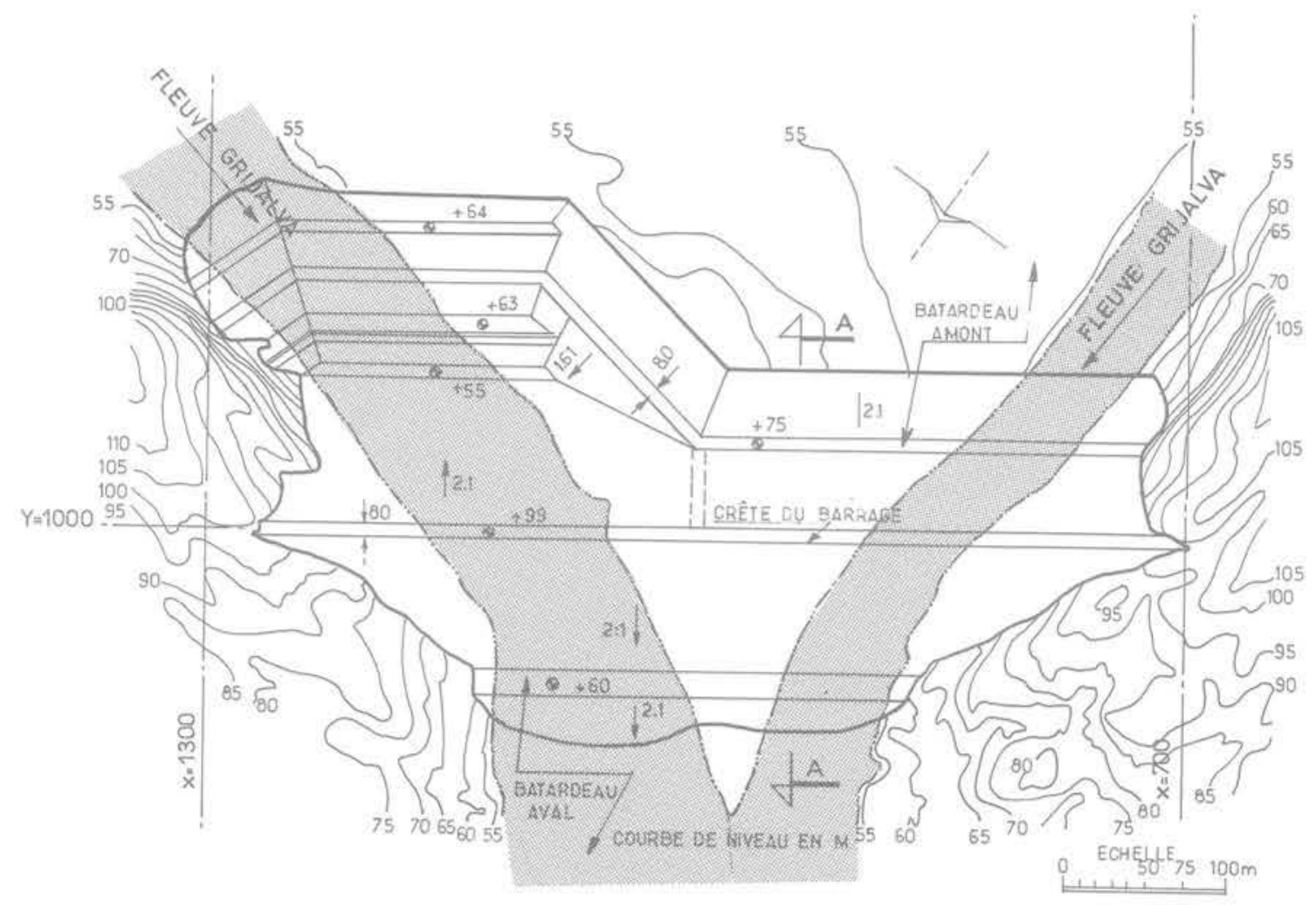

Fig. 3. - Plan du projet avec repérage de la coupe $A A$.
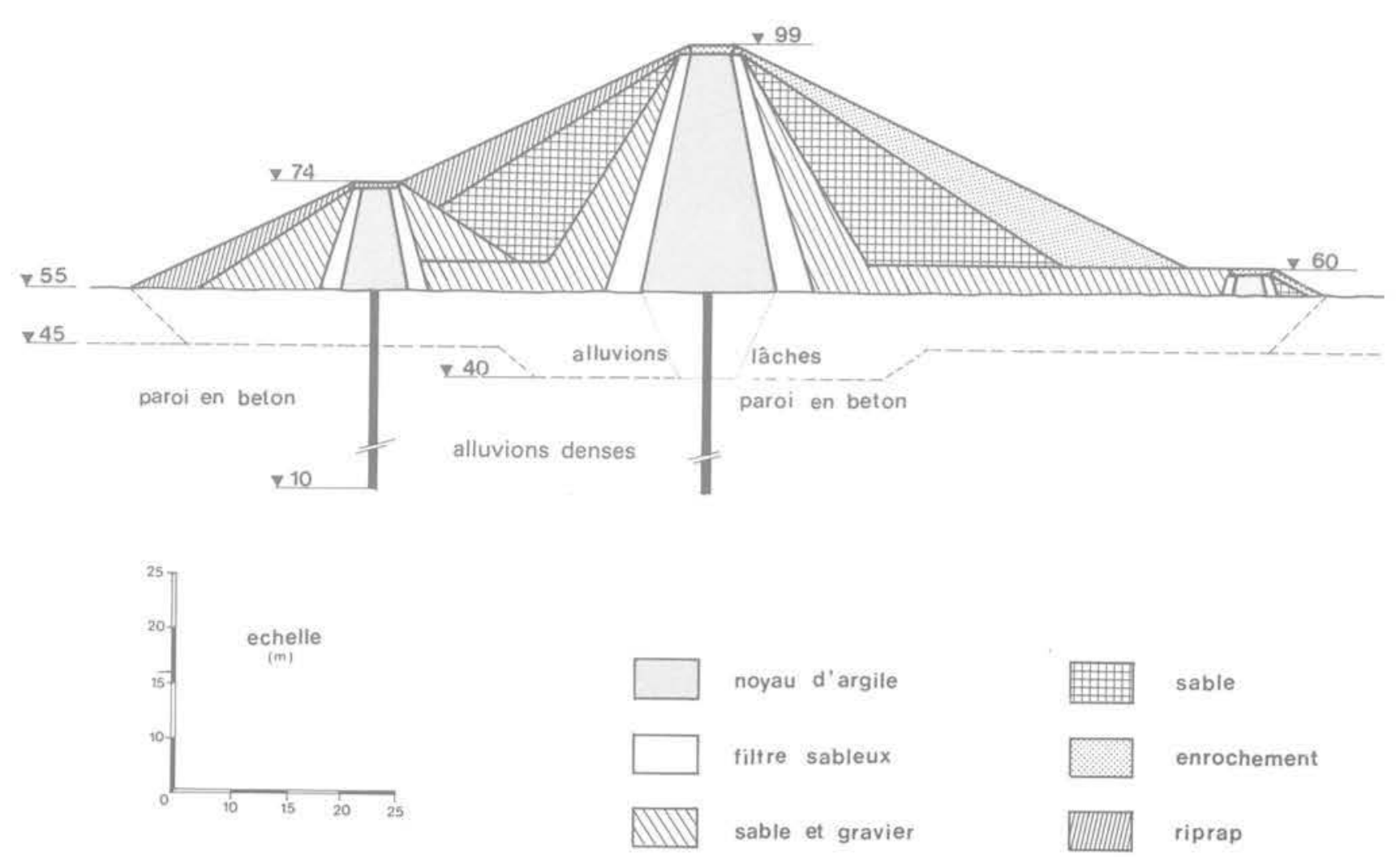

Fig. 4. - Coupe schématique AA du projet. 


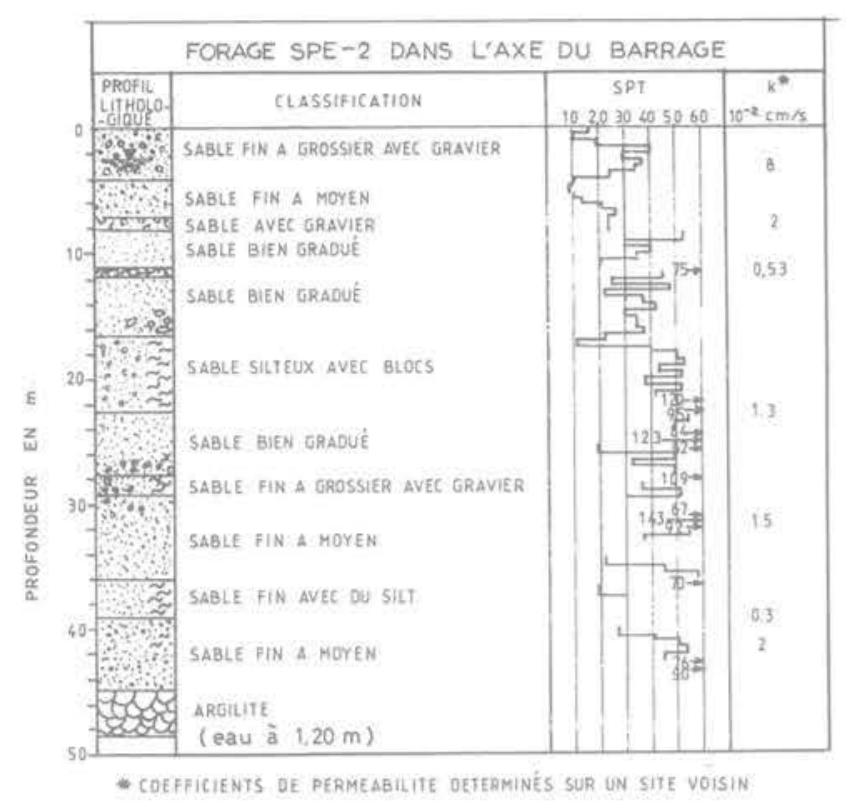

Fig. 5. - Coupe de sondage type (dans le centre du futur barrage).

\section{2. ÉTUDE DU PROJET DE FONDATION}

En raison de la présence de ces lentilles d'alluvions lâches mises en évidence pendant la campagne de reconnaissance de sol, il apparut vite que pour:

- minimiser les tassements différentiels susceptibles de provoquer des fissures de traction dans le noyau,

- assurer la stabilité des talus dans les zones de faible rêsistance au cisaillement,

- diminuer les percolations localement très élevées,

- réduire le potentiel de liquéfaction dans cette zone de séismicité assez élevée,

la tranche supérieure de ces matériaux devait être l'objet d'une densification. Une solution consistait à excaver les alluvions et à les recompacter par couches selon les techniques routières, c'est-à-dire au sec, à l'intérieur d'une enceinte étanche difficile à réaliser en raison des fortes perméabilités locales. Cette solution étant très chère, les méthodes de traitement in situ par moyens mécaniques furent étudiées et la solution de la consolidation dynamique Menard (GAMBIN 1983, 1985) fut retenue comme apportant toute garantie sur le plan technique et ayant le meilleur rapport qualité prix.

\section{RÔLE DE LA CONSOLIDATION DYNAMIQUE DANS LA RÉDUCTION DU POTENTIEL DE LIQUÉFACTION}

S'il est devenu courant de savoir que la consolidation dynamique en augmentant la densité d'un sol permet d'augmenter sa résistance au cisaillement, de réduire son coefficient de perméabilité, et de diminuer les risques de tassements différentiels du futur ouvrage, on connaît moins son intérêt dans la réduction du potentiel de liquéfaction (GAMBIN et autres, 1979).

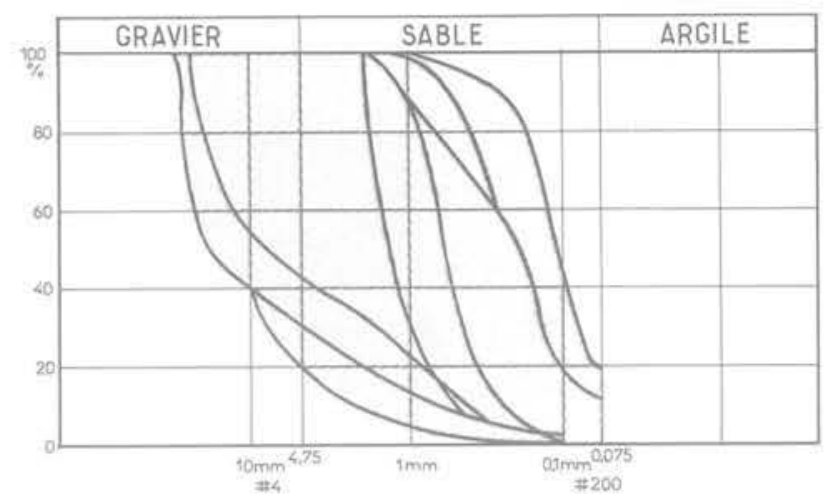

ECHANTILLON AU CARROTIER FENDU

Fig. 6. - Exemples et fuseau des courbes granulométriques des alluvions à traiter.

Les facteurs pris en compte dans la détermination du potentiel de liquéfaction (SEED, 1976) sont:

- la densité relative du matériau en fonction de la profondeur;

- le coefficient de pression horizontale des terres au repos $\mathrm{K}_{\mathrm{o}}$;

- l'histoire du sol;

- la forme des grains et la texture du sol;

- la durée et l'amplitude du train d'onde sismique.

Or la consolidation dynamique permet d'agir sur les trois premiers facteurs, les seuls modifiables (encore que l'on puisse constater une cimentation des grains dans les couches supérieures traitées lorsqu'il s'agit de matériaux typiquement pulvérulents).

Nous ne reviendrons pas sur l'action évidente sur la densité relative. L'accroissement du coefficient de pression horizontale des terres au repos $\mathrm{K}_{0}$ est moins souvent considéré, mais d'une part, la plupart des méthodes de compactage mécanique (y compris les rouleaux sur des couches d'épaisseur limitée) provoquent l'accroissement de $\mathrm{K}_{\mathrm{o}}$, d'autre part ce phénomène, qui se manifeste sur toute l'épaisseur de la couche traitée, peut être mesuré facilement à l'aide de capteurs de pression totale (MENARD, 1974) et confirmé par les valeurs souvent élevées du rapport $\mathrm{E} / \mathrm{pl}$ mesurées au pressiomètre: avant consolidation dynamique la valeur est inférieure à 10 , après traitement elle est supérieure à 15 .

L'histoire d'un sol, c'est-à-dire l'histoire de la succession chronologique des champs de contraintes et déformations statiques et dynamiques auxquels a été soumis le sol depuis son dépôt a une grande influence sur son comportement aux séismes ultérieurs.

Il a été démontré (SEED et autres, 1977) que la susceptibilité d'un sol à la liquéfaction diminue lorsque 
le sol a déjà été mis en liquéfaction avec des déformations de cisaillement faible et que ses grains ont été réarrangés vers une densité plus élevée.

Des expériences au laboratoire de l'Ecole Centrale sur un appareil triaxial cyclique (BIAREZ et autres, 1976) ont montré que le nombre de cycles nécessaires pour provoquer la liquéfaction d'un matériau densifié par cycles de liquéfaction et drainage était bien supérieur à celui requis pour le même matériau densifié par serrage statique, quand bien même l'augmentation de densité dans le premier cas pouvait apparaître nettement plus faible (fig. 7).

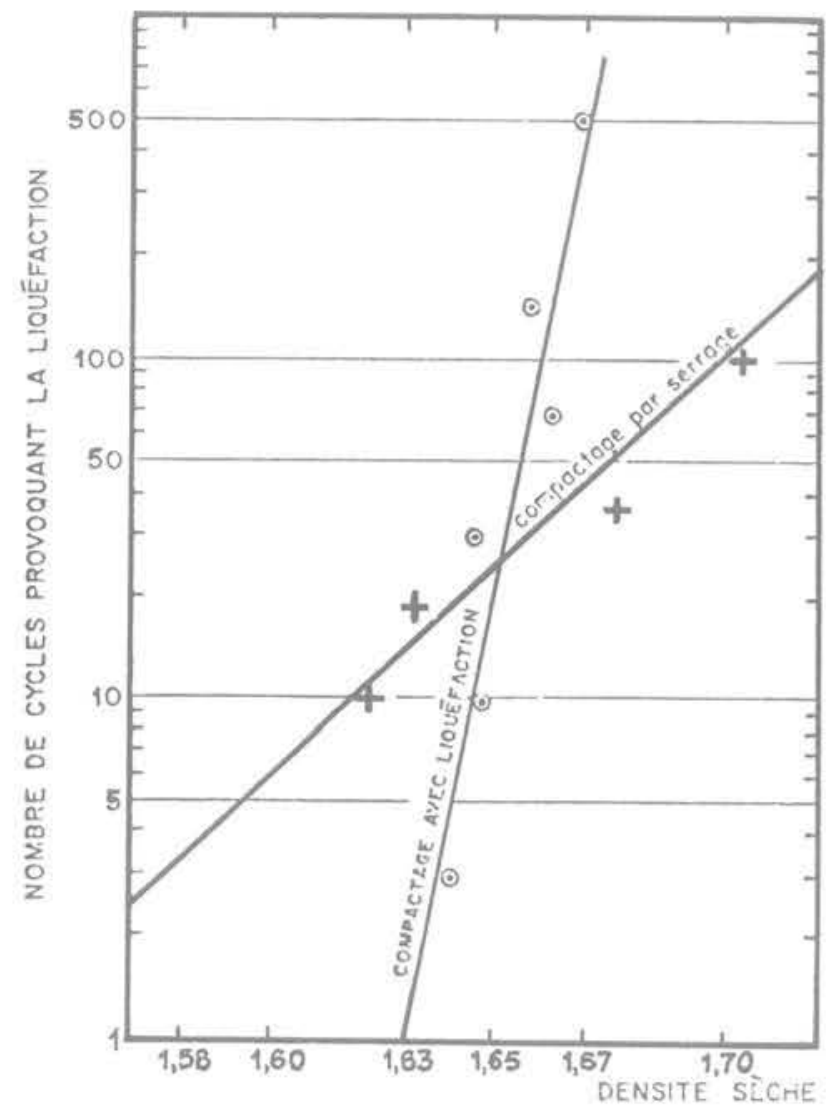

Fig. 7. - Comparaison entre les variations des potentiels de liquéfaction (mesurés en nombre de cycles nécessaires pour atteindre la liquéfaction) d'échantillons densifiés soit par serrage isotrope, soit par cycles successifs de liquéfaction et drainage.

On remarque de même in situ qu'à chaque nouvelle passe de consolidation dynamique le degré de liquéfaction obtenu pour la même énergie ne cesse de décroître.

A titre d'information complémentaire et bien qu'on n'ait pas à demander à la consolidation dynamique de pré-créer des excitations similaires à celles du tremblement de terre pour lequel l'ouvrage est calculé, on a représenté sur la figure 8 les spectres comparés des effets d'un impact de $30000 \mathrm{~kJ}$ relevés à $28 \mathrm{~m}$ du point de chute et de certains séismes récents.

\section{CAHIER DES CHARGES DU MARCHÉ}

Compte tenu des essais de liquéfaction en vraie grandeur des alluvions du Grijalva réalisés à l'occasion de la construction du barrage de Netzahuacoyotl à l'aide de tirs d'explosifs, il avait été possible d'imposer les conditions de réception suivantes pour les matéraux traités (fig. 9):

- densité relative supérieure à $70 \%$ jusqu'à $7 \mathrm{~m}$ de profondeur;

- densité relative supérieure à $60 \%$ de 9 à $15 \mathrm{~m}$ de profondeur.

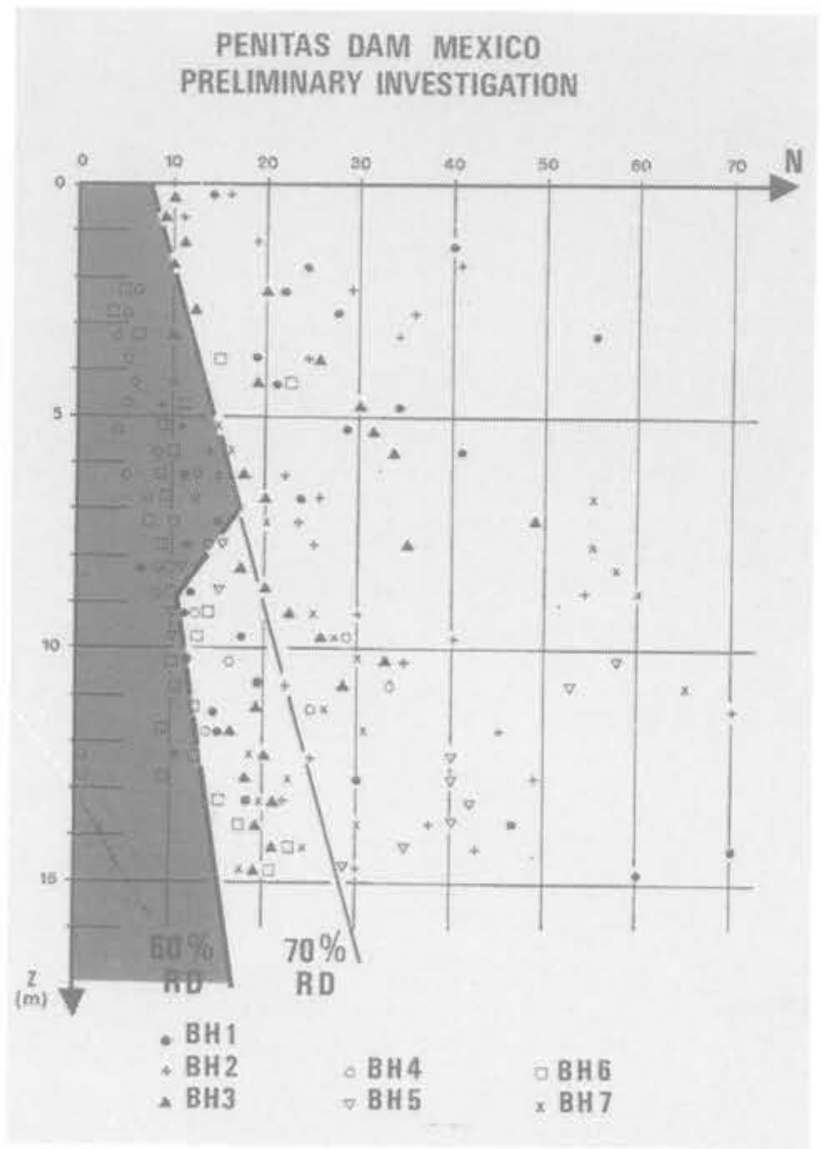

Fig. 9. - Calage par rapport aux densités relatives à obtenir des résultats des essais S.P.T. avant traitement.

\section{RÉALISATION DES TRAVAUX}

Les travaux furent confiés par le Maître d'Ouvrage C.F.E. à C.I.M.E.S.A. la filiale mexicaine de Soletanche, C.I.M.E.S.A. s'adjoignit l'assistance de Techniques Louis MENARD S.A. pour mener les opérations à bonne fin.

Compte tenu de la profondeur de la couche à traiter, $15 \mathrm{~m}$, il était nécessaire que les passes destinées à densifier les couches profondes permettent de dévelop- 


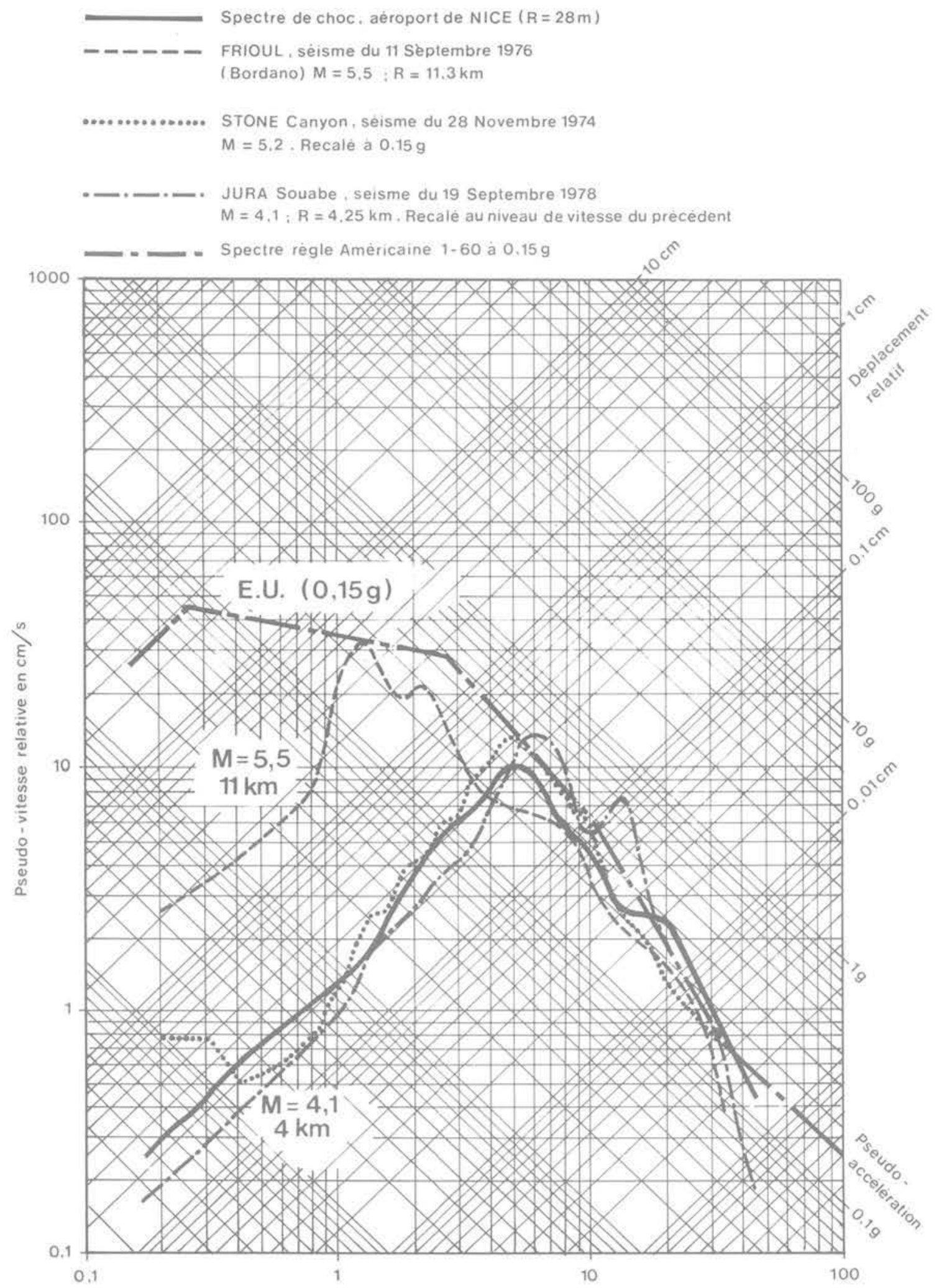

Fig. 8. - Comparaison d'un spectre des vibrations verticales mesurées à faible profondeur à $28 \mathrm{~m}$ d'un impact de $30000 \mathrm{~kJ}$ et de spectres de séismes récents. 
per une énergie de $10 \times(2 \times 15)^{2}$ ou $9000 \mathrm{~kJ}$, ce qui a conduit à utiliser un tripode de conception MENARD levant un pilon de $370 \mathrm{kN}$ de $6,25 \mathrm{~m}^{2}$ de surface de base et constitué de plaques d'acier boulonnées entre elles (fig. 10), tombant en chute libre d'une hauteur de $27 \mathrm{~m}$. De plus, pour les passes destinées à compacter les niveaux supérieurs une grue Bucyres-Erie 88 permettait de lever un pilon de $150 \mathrm{kN}$ tombant de $20 \mathrm{~m}$.

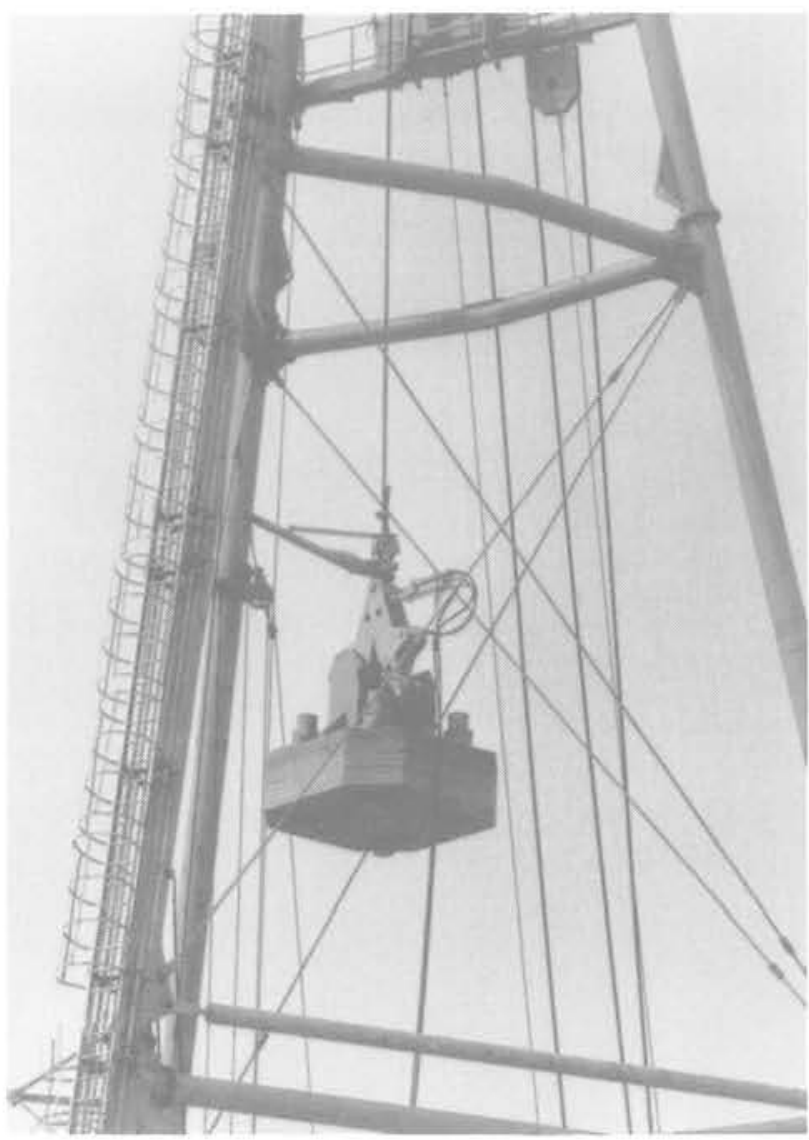

Fig. 10. - Vue partielle du tripode permettant de détailler le pilon retenu par sa pince hydraulique pendant son levage.

Ces conditions s'appliqueraient non seulement aux alluvions en place mais également aux remblais sableux disposés:

- sur $3 \mathrm{~m}$ d'épaisseur après décapage sur les zones émergeantes;

- sur $6 \mathrm{~m}$ d'épaisseur après dragage des limons dans le lit mineur des bras du fleuve.

Nous n'allons présenter icí que la tranche de travaux de 1981 correspondant au traitement de la zone entre les deux bras du fleuve ainsi que la zone du bras gauche, l'ensemble correspondant à une surface de $56000 \mathrm{~m}^{2}$. La deuxième tranche, portant la surface totale à $82000 \mathrm{~m}^{2}$ a été traitée au printemps 1984 .

Sur les figures 11 et 12 on voit une vue d'ensemble du site et quelques aspects particuliers du chantier.

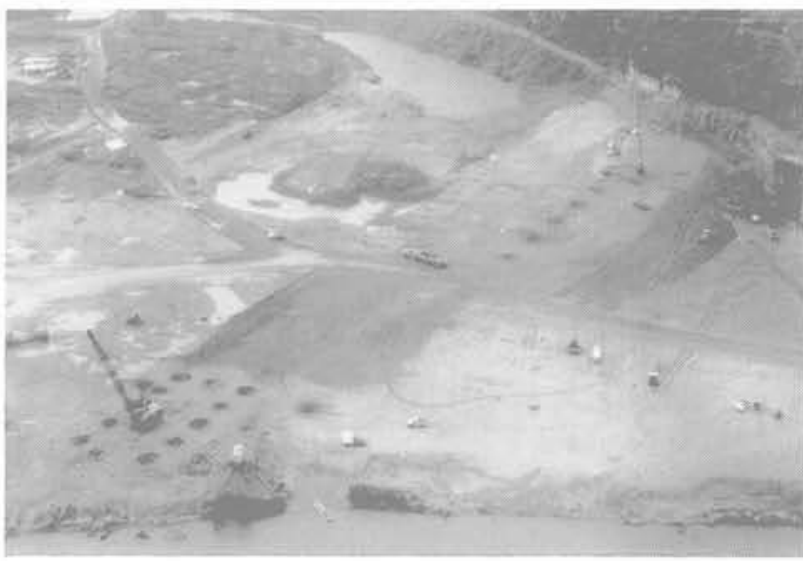

Fig. 11. - Vue générale du site: au premier plan le bras de droite du Grijalva, puis l'atelier de consolidation dynamique avec une grue, enfin l'atelier du tripode sur le bras de gauche du fleuve remblayé.

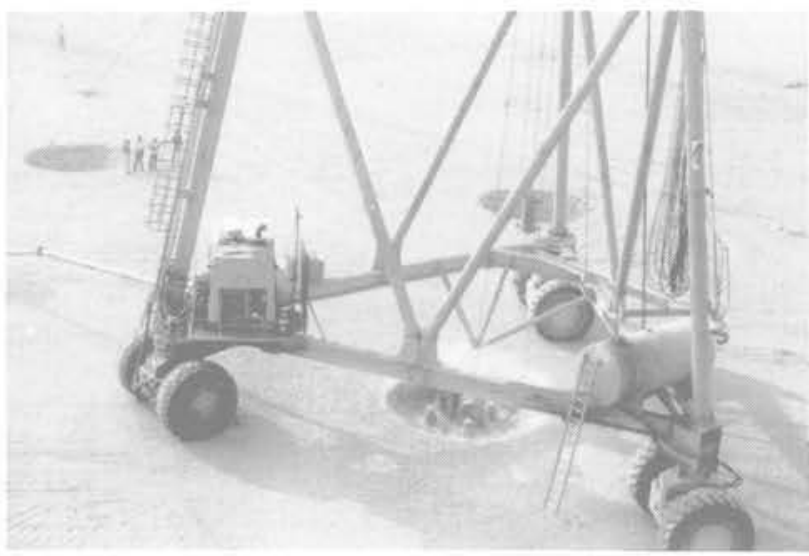

Fig. 12. - Vue rapprochée de la base du tripode après la chute du pilon qu'on apercoit dans le cratère entre les trois couples de rove. L'échelle d'un cratère est donnée par le groupe de personnages en haut à gauche.

Sur le tableau I sont rassemblées les caractéristiques de chaque passe, sachant que la zone centrale de $70 \mathrm{~m}$ de large devait subir un traitement d'énergie supérieur. On trouvera sur le plan de la figure 13 l'emplacement des différents cratères pour les 3 premières passes, la quatrièrne passe étant exécutée à empreintes jointives de manière à supprimer toute hétérogénéité de surface (sauf sur $1 \mathrm{~m}$ d'épaisseur).

\section{SUIVI GÉOTECHNIQUE}

Comme pour chaque chantier de consolidation dynamique un suivi strict de la réaction du sol aux impacts fut respecté:

- mesure des volumes des cratères: 
Tableau I: Caractéristique des différentes passes

\begin{tabular}{|c|c|c|c|c|c|}
\hline$N^{\circ}$ de passe & Matériel & $\begin{array}{l}\text { Energie par } \\
\text { coup (kJ) }\end{array}$ & Maille (m) & $\begin{array}{l}\text { Energie totale } \\
\qquad \mathrm{kJ} / \mathrm{m}^{2}\end{array}$ & $\begin{array}{c}\text { Tassement } \\
\text { moyen }(\mathrm{mm})\end{array}$ \\
\hline $\begin{array}{c}\text { Zone centrale } \\
1 \\
2 \\
3 \\
4\end{array}$ & $\begin{array}{c}\text { Tripode } \\
\text { Tripode } \\
\text { Grue } \\
\text { Grue }\end{array}$ & $\begin{array}{r}10000 \\
10000 \\
3000 \\
3000\end{array}$ & $\begin{array}{r}20 \\
20 \\
14 \\
2\end{array}$ & $\begin{array}{l}500 \\
350 \\
350 \\
750\end{array}$ & $\begin{array}{l}64 \\
43 \\
49 \\
67\end{array}$ \\
\hline \multicolumn{4}{|c|}{ 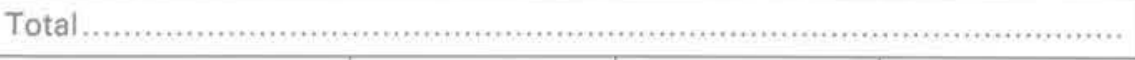 } & 1950 & 223 \\
\hline $\begin{array}{c}\text { Zone amont et aval } \\
1 \\
2 \\
3 \\
4\end{array}$ & $\begin{array}{c}\text { Tripode } \\
\text { Tripode } \\
\text { Grue } \\
\text { Grue }\end{array}$ & $\begin{array}{r}10000 \\
10000 \\
3000 \\
3000\end{array}$ & $\begin{array}{r}20 \\
20 \\
14 \\
2\end{array}$ & $\begin{array}{l}500 \\
300 \\
250 \\
750\end{array}$ & $\begin{array}{l}64 \\
43 \\
31 \\
67\end{array}$ \\
\hline \multicolumn{4}{|c|}{ Total } & 1800 & 205 \\
\hline
\end{tabular}

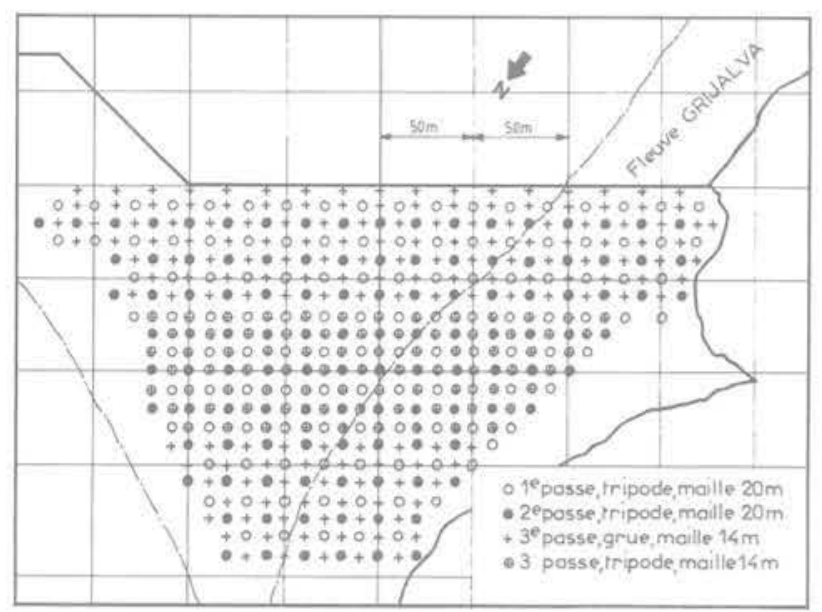

Fig. 13. - Plan des points d'impacts des trois premières passes.

- mesure des surpressions interstitielles:

- mesure de l'amélioration des caractéristiques mécaniques entre chaque passe par essais in situ.

Les volumes des cratères servirent à estimer l'enfoncement moyen de chaque nceud de la grille de compactage, enfoncement qui est ensuite confirmé par un nivellement général de la plateforme après remblaiement des cratères au bouteur.

Les capteurs de pressions interstitielles, du type hydraulique, à circuit fermé, montrèrent tous que les surpressions se dissipaient à $90 \%$ dans l'heure qui suivait l'application des impacts, ce qui a permis plus de liberté et de rapidité dans la conduite du chantier.

A la fois C.IM.E.S.A. et C.F.E. procédèrent à des essais in situ après s'être étalonnés sur des essais complémentaires avant traitement, réalisés:

- au pressiomètre;

- au pénétromètre dynamique S.E.R.M.E.S.;

- au pénétromètre dynamique C.F.E. selon la rêpartition donnée au tableau II en ce qui concerne les essais de l'entrepreneur.

Tableau II: Répartition des sondages de contrôle de l'entrepreneur

\begin{tabular}{|l|c|c|c|}
\hline & $\begin{array}{c}\text { Avant } \\
\text { travaux }\end{array}$ & $\begin{array}{c}\text { Pendant } \\
\text { travaux }\end{array}$ & $\begin{array}{c}\text { Après } \\
\text { travaux }\end{array}$ \\
\hline Pressiomètre ............ & 8 & 4 & 11 \\
\hline $\begin{array}{l}\text { Pénétromètre } \\
\text { dynamique SERMES }\end{array}$ & 4 & - & 24 \\
\hline
\end{tabular}

On a reporté sur les figures 14 et 15 les valeurs moyennes mesurées avant et après traitement pour l'ensemble du site.

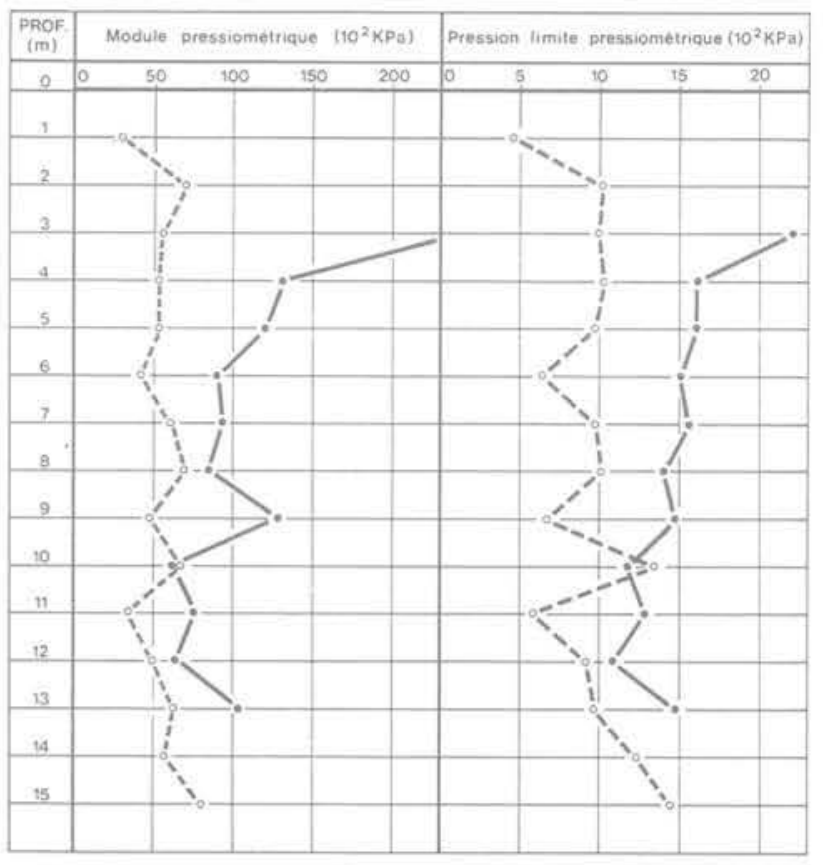

- - - - Avant consolidation dynamique - Apres consolidation dynamique

Fig. 14. - Valeurs moyennes de E et PI mesurées au pressiomètre avant et après traitement. 


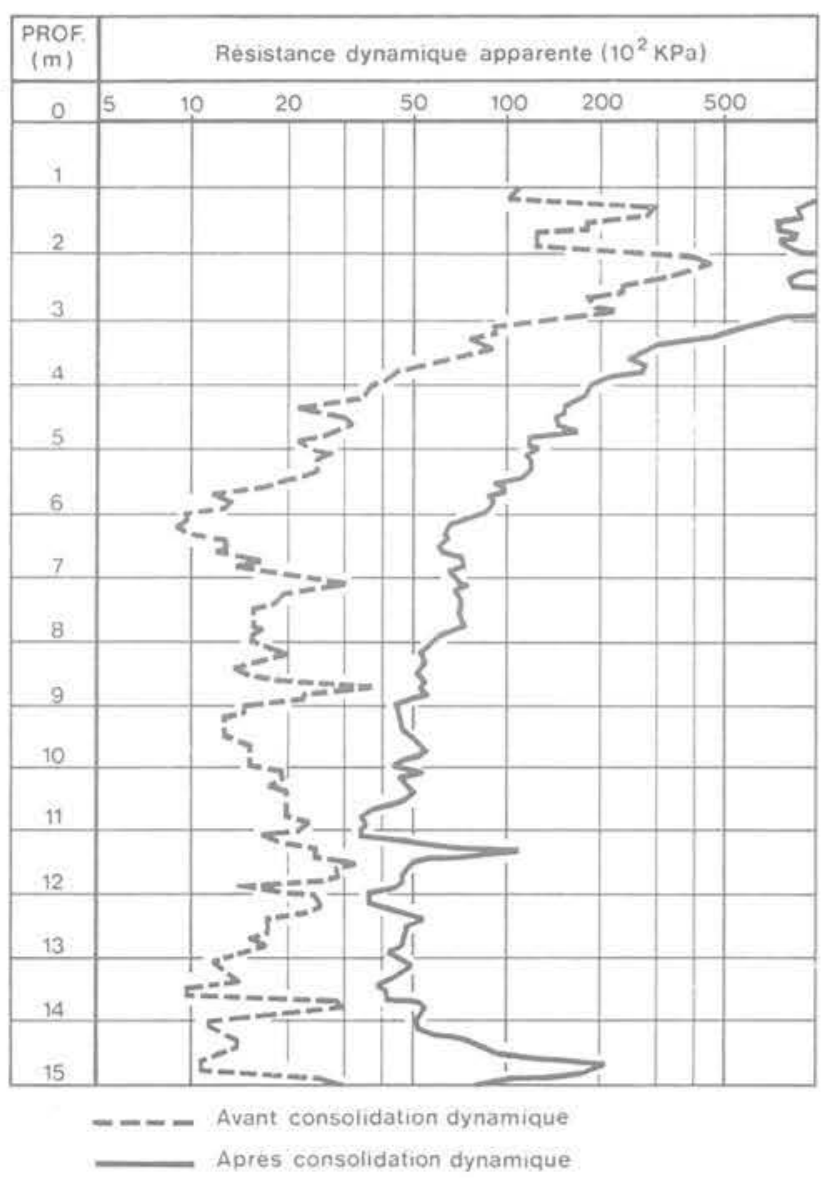

Fig. 15. - Valeurs moyennes des résistances à la pénétration dynamique (S.E.R.M.E.S.) avant et après traitement (échelle logarithmique).

Il fut constaté que toutes les valeurs faibles de caractéristiques mécaniques mesurées avant traitement avaient disparu, à l'exception de celles fournies par un résultat dans le sondage P.M.I.-4, forage situé d'ailleurs en bordure de la zone traitée. Toutefois les pénétromètres PD16-4, PD17-4 et PD18-4 exécutés au voisinage n'ayant pas mis en évidence d'anomalie, il en fut déduit qu'il s'agissait d'une lentille argileuse très limitée, peut-être en surpression interstitielle.

Plusieurs traitements statistiques peuvent être exécutés sur les résultats obtenus avant traitement et ceux obtenus après:

- comparaison des moyennes de tous les essais à une profondeur donnée; ainsi, sur les 5 premiers mètres, l'augmentation du module pressiométrique est de $250 \%$ et celle de la pression limite d'au moins $100 \%$ (compte tenu du fait que les essais pressiométriques n'ont pas été poussés au-delà de $2,5 \mathrm{MPa}$ ). De 5 à $15 \mathrm{~m}$ de profondeur, les résultats montrent des augmentations de $60 \%$ sur les modules et $45 \%$ sur les pressions limites. Sur les résistances dynamiques de pointes, on observe des augmentations encore plus spectaculaires: $350 \%$ jusqu'à $5 \mathrm{~m}, 250 \%$ entre 5 et $15 \mathrm{~m}$, ce qui s'explique par la relation exponentielle entre cette résistance et l'angle de frottement interne; - comparaison des prévisions de comportement du sol au droit de chaque sondage avant et après traitement. Ainsi, le tassement prévisible est-il divisé par 1,8 et le tassement différentiel par plus de 2,2.

Ces rapports d'amélioration peuvent apparaître assez faibles, par rapport aux performances obtenues sur d'autres chantiers, mais d'une part, l'énergie appliquée au mètre cube a été réduite (moyenne 120 à $130 \mathrm{kN} \times \mathrm{m}$ ), d'autre part, l'ensemble des alluvions n'était pas globalement médiocre avant traitement.

\section{CONCLUSION}

La consolidation dynamique s'est avérée efficace pour améliorer les 15 premiers mètres d'alluvions formant le terrain de fondation du futur barrage de Pénitas. Les lentilles de matériaux lâches mais toujours à faible pourcentage de fines ont été convenablement densifiées. Les horizons déjà très denses ont, par contre, été peu affectés ou même ont pu être légèrement décompactés, entraînant une meilleure homogénéisation globale.

\section{BIBLIOGRAPHIE}

J. BIAREZ, M. RUDELLE. - Comportement aux séismes des sables fins, rapport de recherche, Ecole Centrale (non publié) 1976.

M. GAMBIN. - La Consolidation Dynamique MENARD, journée d'étude du 28 avril 1982, Annales I.T.B.T.P., oct. 1983.

M. GAMBIN. - Dix ans de consolidation dynamique, Annales I.T.B.T.P. (à paraître) en 1985.

M. GAMBIN, J.F, CAPELLE et J. DUMAS. - La Consolidation Dynamique, une technique permettant de diminuer les risques de liquéfaction des sols fins saturés en cas de tremblement de terre, $3^{\mathrm{e}}$ Congrès Canadien de Génie Sismique, McGILL, Montréal, 1979.

L. MENARD, - Un procédé de consolidation, le pilonnage intensif, Annales I.T.B.T.P., Sept. 1974.

H.B. SEED. - Evaluation of Soil Liquefaction. Effects on Level Ground during Earthquake, dans "Liquefaction Problems in Geotechnical Engineering * A.S.C.E. Preprint 2592, New York 1976.

H.B SEED, K. MORI \& C. CHAN. - Influence of Seismic History on Liquefaction of Sands, G.T. Journal n4, A.S.C.E, avril 1977. 